\title{
A case of atypical progressive multifocal encephalopathy mimicking acute ischemic stroke: case report and review of literature
}

\author{
Maria Teresa Infante', Giovanni Novi'1,2, Riccarda Gentile', Laura Malfatto', Lucio Castellan³, Carlo Serrati', \\ Laura Barletta ${ }^{3}$
}

${ }^{\prime}$ Neurology Unit, Department of Neurosciences, IRCCS San Martino IST, Largo Rosanna Benzi 10, 16132 Genoa, Italy.

${ }^{2}$ Department of Neurology, Ophtalmology, Genetics, Maternal and Child Health, University of Genoa, Largo Daneo 3, 16132 Genoa, Italy.

${ }^{3}$ Neuroradiology Unit, IRCCS San Martino IST, Largo Rosanna Benzi 10, 16132 Genoa, Italy.

Correspondence to: Dr. Maria Teresa Infante, Neurology Unit, Deparment of Neurosciences, IRCCS San Martino IST, Largo Rosanna Benzi 10, 16132 Genoa, Italy. E-mail: infante.mariateresa@gmail.com

How to cite this article: Infante MT, Novi G, Gentile R, Malfatto L, Castellan L, Serrati C, Barletta L. A case of atypical progressive multifocal encephalopathy mimicking acute ischemic stroke: case report and review of literature. Neuroimmunol Neuroinflammation 2017;4:211-5.

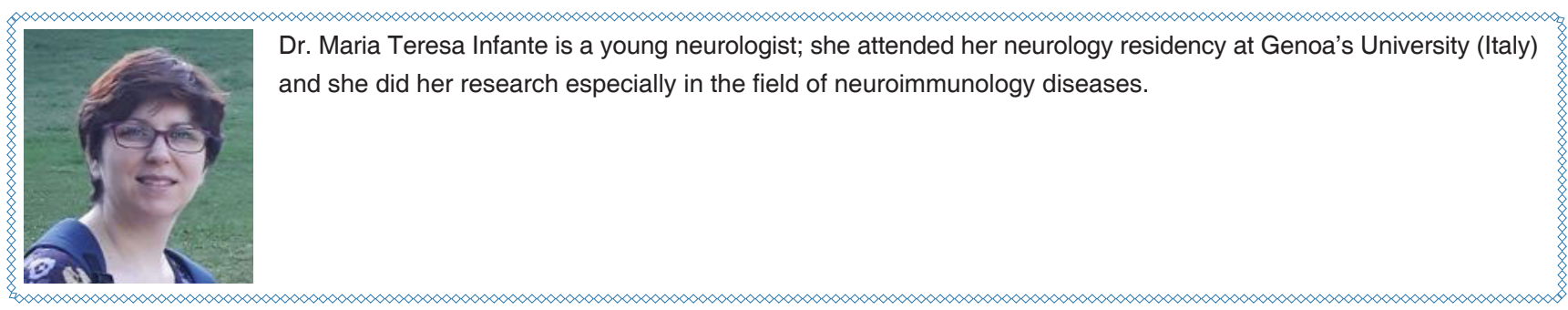

Article history:

Received: 25 Nov 2016

Accepted: 10 Jul 2017

Published: 19 Oct 2017

Key words:

Progressive multifocal

encephalopathy,

rituximab,

immunosuppression

\section{ABSTRAC T}

Progressive multifocal encephalopathy (PML) is a rare but often fatal infectious brain disease caused by the reactivation of John Cunningham polyomavirus. Reactivation occurs in immunocompromised individuals with AIDS and leukemia, on chemotherapy or being treated with immunosuppressant drugs (e.g. monoclonal antibodies). Cases of PML have been described in patients treated with natalizumab, efalizumab and rituximab used, respectively, for the treatment of (1) multiple sclerosis, (2) psoriasis and (3) haematological malignancies or systemic autoimmune diseases (rheumatoid arthritis and systemic lupus erythematosus). The authors describe an unusual case of acute brainstem and cerebellar PML following chemotherapy for chronic lymphatic leukemia diagnosed 4 years before the onset of PML in a 75-year-old man. The patient was treated with high dose chemotherapy and rituximab with complete response. The onset of symptoms of PML was very rapid and occurred after more than two years from last rituximab infusion; patient had a sudden neurological deterioration, with rapid progression to death in about a month from the onset of symptoms. Lesions were localized in the cerebellum, brainstem and such pattern has been reported in very few cases in the literature.

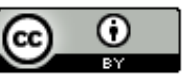

This is an open access article licensed under the terms of Creative Commons Attribution 4.0 International License (https://creativecommons.org/licenses/by/4.0/), which permits unrestricted use, distribution, and reproduction in any medium, as long as the original author is credited and the new creations are licensed under the identical terms.

For reprints contact: service@oaepublish.com

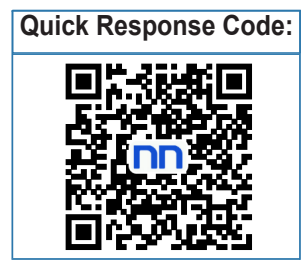




\section{INTRODUCTION}

Progressive multifocal encephalopathy (PML) is a demyelinating infectious disease of the central nervous system caused by reactivation of John Cunningham polyomavirus (JCV) and often leads to death resulting from progressive oligodendrocytes infection and lysis ${ }^{[1]}$. Prior to human immunodeficiency virus era, this infection was seen in severely immunosuppressed patients, including individuals with hematological malignancies, organ transplantation or chronic inflammatory conditions, such autoimmune disorders, with an incidence of 4 cases $/ 100,000^{[2-4]}$.

Clinical presentation is heterogeneous: PML usually begins as a subacute illness that typically evolves from focal or multifocal neurological deficits progressing over days to weeks, leading to severe disability and, ultimately, to death. In some cases focal neurological syndromes may present acutely and can be mistaken for stroke. The ratio of cerebral to brainstem involvement is estimated approximately to be 10:1. For reasons that are unclear, brainstem involvement is more common in acquired immunodeficiency syndrome patients, with a ratio of approximately $4: 1^{[1]}$.

Diagnosis of PML is clinical and radiological, principally magnetic resonance imaging (MRI) based; diagnosis is then confirmed by demonstration of JCV DNA in the cerebrospinal fluid by polymerase chain reaction (PCR) test ${ }^{[4,5]}$.

Key MRI diagnostic features are: (1) cortical and deep cerebellar nuclei (in infratentorial involvement) sparing; (2) absence of mass effect on subarachnoid or ventricular spaces and on adjacent areas; and (3) lack of contrast enhancement (CE). Diffusionweighted images (DWI) sequences are also able to assess the extension of white matter lesions; regions with increased DWI intensity represent white matter areas characterized by reduced water diffusion due to cytotoxic edema ${ }^{[2-6]}$.

Demyelination is usually multifocal, involving hemispheric white matter (parietal, frontal and occipital lobes) and/or cerebellar peduncles. In literature, rare cases of posterior fossa localization are described ${ }^{[7]}$.

To date, there is no established therapy for PML and the treatment is mostly supportive ${ }^{[8]}$.

\section{CASE REPORT}

The patient was a 75-year-old male with a previously (2012) diagnosed chronic lymphocytic leukemia.
He was treated with chlorambucil in 2012, followed by 6 cycles of fludarabine, cyclophosphamide and lenalidomide in 2013 , with complete response; he was then treated with rituximab and steroids for hemolytic anemia (for 4 weeks in 2014) with complete regression.

In March 2016, he was admitted to the emergency department for acute dizziness and ataxia followed, after one day, by dysarthria and left limbs ataxia. A cerebral unenhanced computed tomography (CT) scan showed diffuse ipodensity of the left cerebellum and the middle cerebellar peduncle without mass effect [Figures 1 and 2].

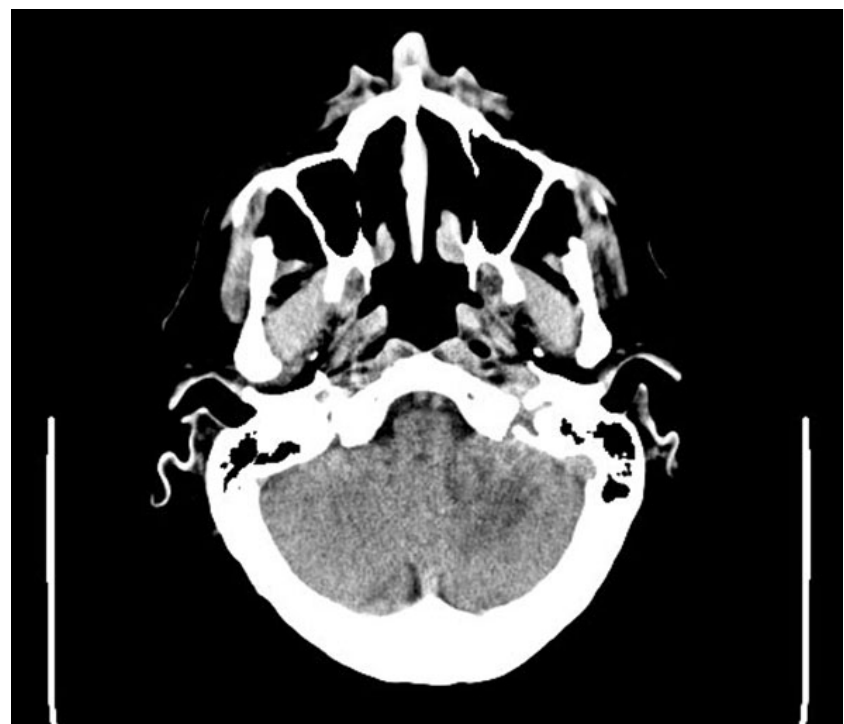

Figure 1: Brain computer tomography scan without contrast showing ipodensity al left cerebellar hemisphere without mass effect

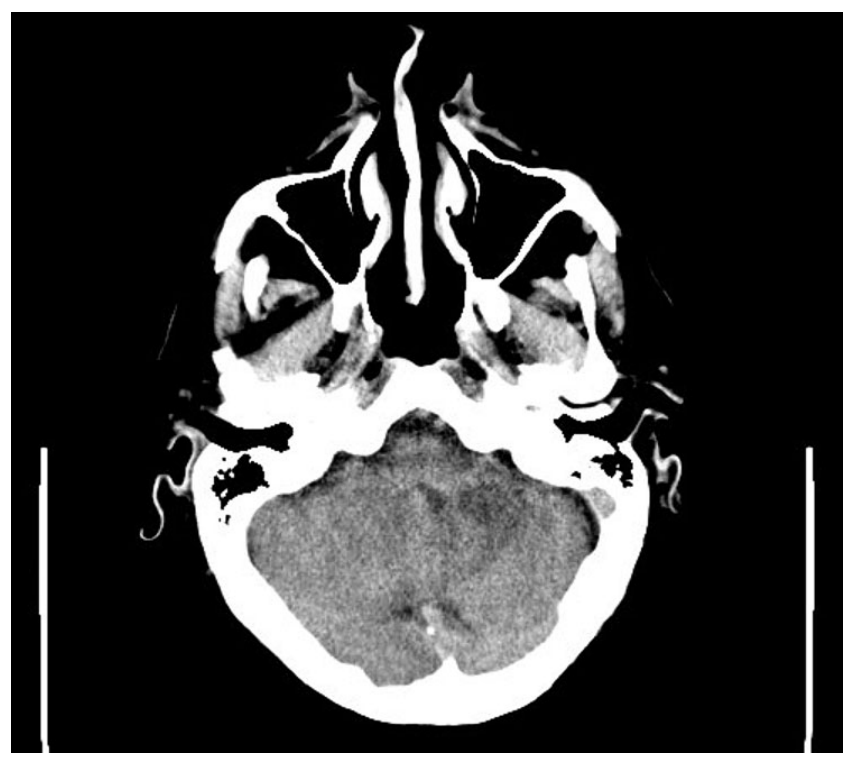

Figure 2: Brain computer tomography scan without contrast showing ipodensity al left cerebellar hemisphere spreading to middle cerebellar peduncle 
Neurological examination showed that the patient had dysarthria and left limbs dysmetria, left-beating nystagmus and balance difficulties. Acute ischemic stroke was suspected and antiplatelets therapy with aspirin was started, with transitory symptoms improvement.

Seven days later he presented with worsening of dizziness, nausea and lack of appetite; a second brain scan CT (unchanged) and gastroenterological investigations (negative) were performed.

The patient was discharged, but 10 days later, he had acute onset of involuntary movements in left arm that were interpreted as partial epileptic seizures. Antiepileptic therapy with levetiracetam was started with good response. Two days later he had a rapid worsening of symptoms, with alteration of consciousness; he was responsive only to pain stimuli, left limbs hyposthenia and left gaze deviation also appeared. A CT scan showed an extension of the brainstem lesion with middle cerebellar peduncle involvement. Such a finding, together with clinical deterioration, suggested the presence of a partial basilar thrombosis/embolism. A CT angiography was then performed, showing patency of basilar and vertebral arteries. CT scan findings were interpreted as caused by a mass effect of the pre-existing lesion instead of a true extension of the lesion itself, due to the intrinsic low contrast resolution of the exam. Due to the presence of fever and rigor nucalis, infective encephalitis was suspected, and a lumbar puncture was performed, showing only mirror pattern oligoclonal

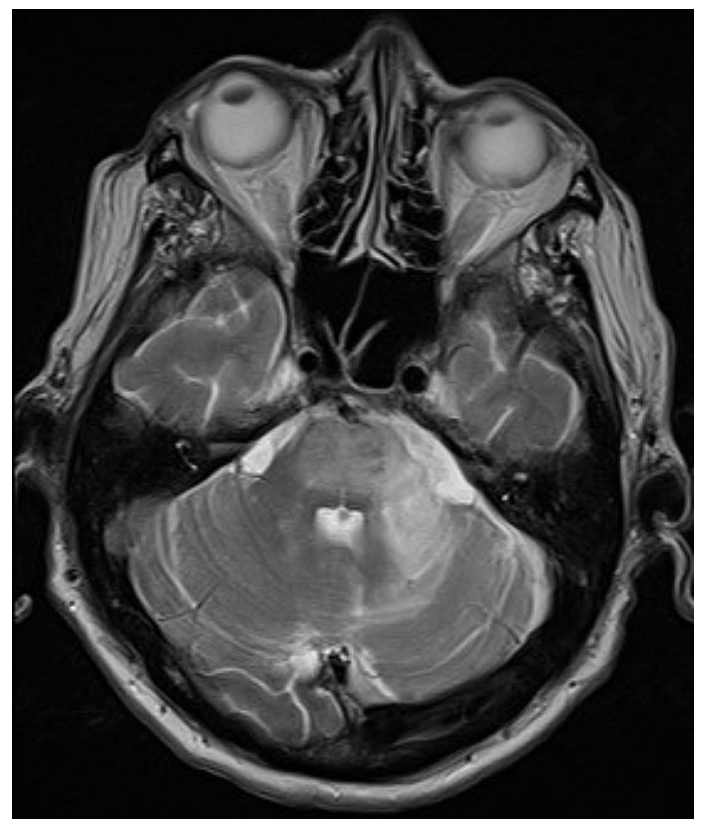

Figure 3: Axial brain magnetic resonance imaging, T2 sequences, revealing asymmetric T2 hyperintensity of the white matter of middle cerebellar peduncles and upper pons without oedema bands on cerebrospinal fluid (CSF) and serum, PCR for neurotropic viruses (HSV, VZV, CMV, EBV, Adenovirus and Enterovirus) and cultural CSF examination were negative. A broad-spectrum antiviral and antibacterial therapy was started without improvement. Blood test exams were normal.

A brain MRI revealed an asymmetric T2/FLAIR hyperintensity in bilateral cerebellar, middle cerebellar peduncles, upper pons and mesenchephalum white matter without oedema, without CE; DWI showed signal increase without detectable apparent diffusion coefficient changes [Figures 3-5].

Another lumbar puncture was performed and PCR for JCV virus on CSF tested positive with 11,300 copies $/ \mathrm{mL}$.

Patient quickly deteriorated and died 10 days after the diagnosis of PML; due to the severity of clinical condition, the rapid progression of symptoms, and the lack of evidence of efficacy of specific therapies, he was treated only with supportive therapy.

\section{DISCUSSION}

Treatment with monoclonal antibodies is a newly identified predisposing factor for PML development. Among monoclonal antibodies those that increase the risk for PML development are natalizumab, efalizumab and rituximab. At present, more than 70 cases of PML have been associated with the use of rituximab, predominantly in patients treated for

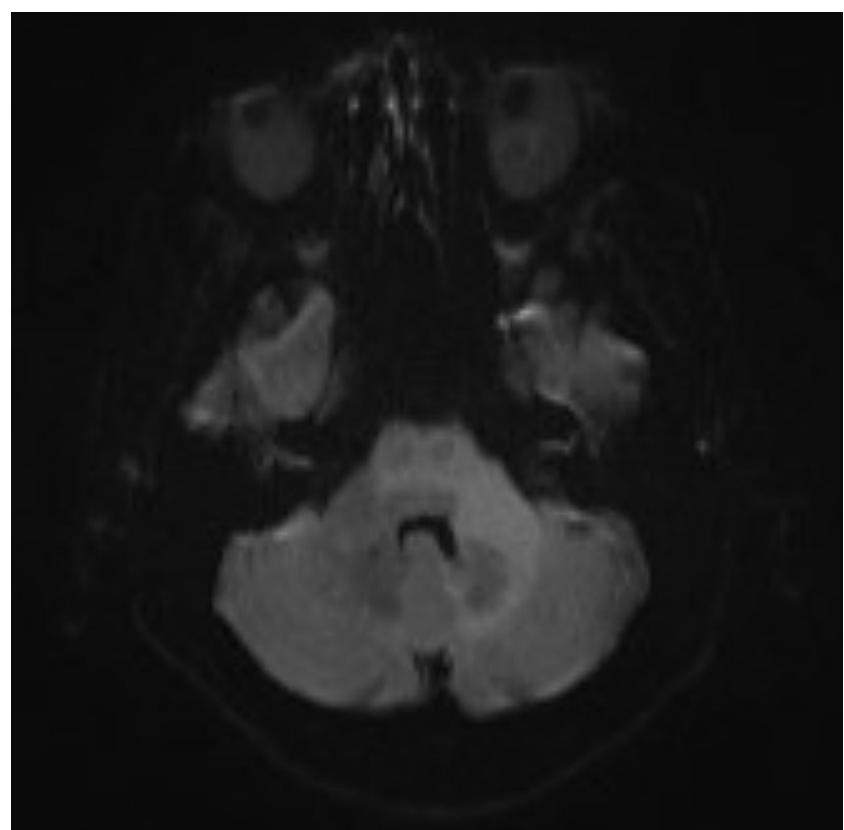

Figure 4: Brain magnetic resonance imaging, diffusion weighted images sequences: increase of signal in the same regions without detectable apparent diffusion coefficient changes 


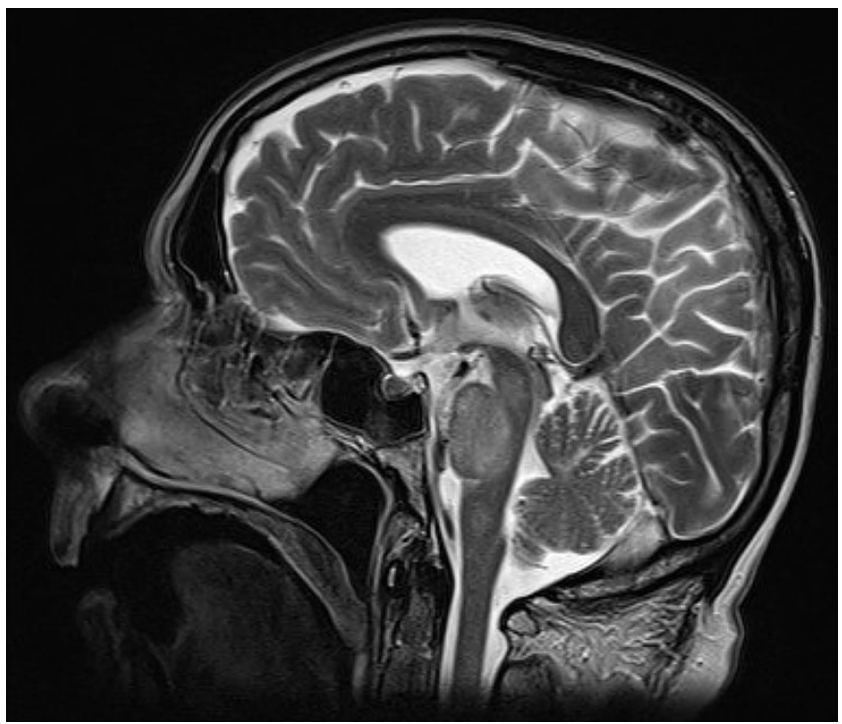

Figure 5: Sagittal brain magnetic resonance imaging, T2 sequences: presence of an asymmetric T2 hyperintensity of white matter in bilateral cerebellar, middle cerebellar peduncles, upper pons and mesenchephalum without oedema

lymphoproliferative disorders. Rituximab is a chimeric human/mouse $\lg 1$ monoclonal antibody that targets CD20 antigen expressed on the surface of both normal and malignant $\mathrm{B}$ lymphocytes; rituximab was approved for the treatment of CD20 positive hematological malignancies, and for non-malignant autoimmune disorders, as rheumatoid arthritis and systemic lupus erythematosus; it can also be used with an "off-label" indication in multiple sclerosis and neuromyelitis optica ${ }^{[7-9]}$.

In general population, the risk of PML is 1 in 200,000 people ${ }^{[7]}$. However, even if the use of rituximab may increase the risk of developing PML, the absolute risk of PML is probably low and does not overcome the benefits in term of mortality in patients with hematological malignancies. The disease in this group of patients appears to set early (following the start of rituximab therapy, with median time of onset from the last dose being about 6 months), with rapid progression and fatal course (median time to death following diagnosis about 2 months). Predisposing factors for rapid progression of the disease include CD4 count $<500$ cells and PML diagnosis within three months following therapy initiation ${ }^{[7-10]}$.

The pathophysiology of the rituximab-PML association is unclear and, as reported in the literature, does not seem to be solely due to a B cells depletion ${ }^{[11,12]}$.

In this case, the association of many atypical characteristics: (1) posterior fossa presentation (as opposed to the classical hemispherical lesions); (2) absence of recent immunosuppression (last rituximab dose administered two years before PML onset) without signs of hematological abnormalities; and (3) rapid neurological deterioration (day-by-day worsening with "sudden" onset of the symptoms) made PML diagnosis particularly tricky. Despite single atypical characteristics have already been reported in the literature, association of such findings have never been reported so far.

As shown by MRI and CT scan, absence of a distinctive vascular territory lesion distribution, despite the acute clinical course and the posterior fossa localization could have been an early clue to a correct diagnosis.

In this case, diagnosis of PML has been delayed because of the clinical presentation (acute onset of cerebellar symptoms), initial lack of anamnestic data (i.e. previous chemotherapies that have been told later) and the presence of hypodense lesion in the first cerebral CT scan, which had been interpreted as acute ischemic lesion, reason why cerebral MRI was not performed in the acute phase.

\section{DECLARATIONS}

\section{Acknowledgments}

The authors thank Dr. Fernando Fronda and Dr. Matteo Pardini for language revision of the manuscript.

\section{Authors' contributions}

Drafted the manuscript: M.T. Infante, G. Novi, L. Barletta

Contributed to the analysis and interpretation of data: L. Malfatto, R. Gentile, L. Castellan, C. Serrati, M.T. Infante, G. Novi, L. Barletta

Revised the manuscript, gave final approval and agreed to be fully accountable for ensuring the integrity and accuracy of the work: M.T. Infante, G. Novi, R. Gentile, L. Malfatto, L. Castellan, C. Serrati, L. Barletta

\section{Financial support and sponsorship None.}

\section{Conflicts of interest}

There are no conflicts of interest.

\section{Patient consent}

The patient and his family gave the informed consent.

\section{Ethics approval}

All the study procedures of this case report were conducted according to the Declaration of Helsinki.

\section{REFERENCES}

1. Sahraian MA, Radue EW, Eshaghi A, Besliu S, Minagar A. Progressive 
multifocal leukoencephalopathy: a review of the neuroimaging features and differential diagnosis. Eur J Neurol 2012;19:1060-9.

2. Yokoyama H, Watanabe T, Maruyama D, Kim SW, Kobayashi Y, Tobinai K. Progressive multifocal leukoencephalopathy in a patient with B-cell lymphoma during rituximab-containing chemotherapy: case report and review of the literature. Int J Hematol 2008;88:443-7.

3. Tan CS, Koralnik IJ. Beyond progressive multifocal leukoencephalopathy: expanded pathogenesis of JC virus infection in the central nervous system. Lancet Neurol 2010;9:425-37.

4. Clavel G, Moulignier A, Semerano L. Progressive multifocal leukoencephalopathy and rheumatoid arthritis treatments. Joint Bone Spine 2017; doi: 10.1016/j.jbspin.2017.03.002.

5. Molloy ES, Calabrese CM, Calabrese LH. The risk of progressive multifocal leukoencephalopathy in the biologic era: prevention and management. Rheum Dis Clin North Am 2017;43:95-109.

6. Ohta K, Obara K, Sakauchi M, Obara K, Takane H, Yogo Y. Lesion extension detected by diffusion-weighted magnetic resonance imaging in progressive multifocal leukoencephalopathy. J Neurol 2001;248:809-11.

7. Felli V, Di Sibio A, Anselmi M, Gennarelli A, Sucapane P, Splendiani A, Catalucci A, Marini C, Gallucci M. Progressive multifocal leukoencephalopathy following treatment with rituximab in an HIVnegative patient with non-Hodgkin lymphoma. A case report and literature review. Neuroradiol J 2014;27:657-64.

8. Lima MA. Progressive multifocal encephalopathy: new concepts. Arq Neuropsiquiatr 2013;71:699-702.

9. Garrote H, de la Fuente A, Oña R, Rodríguez I, Echevarría JE, Sepúlveda JM, García JF. Long-term survival in a patient with progressive multifocal leukoencephalopathy after therapy with rituximab, fludarabine and cyclophosphamide for chronic lymphocytic leukemia. Exp Hematol Oncol 2015;4:8

10. Chakraborty S, Tarantolo SR, Treves J, Sambol D, Hauke RJ, Batra SK. Progressive multifocal leukoencephalopathy in a HIV-negative patient with small lymphocytic leukemia following treatment with rituximab. Case Rep Oncol 2011;4:136-42.

11. Carson KR, Focosi D, Major EO, Petrini M, Richey EA, West DP, Bennett CL. Monoclonal antibody-associated progressive multifocal leucoencephalopathy in patients treated with rituximab, natalizumab, and efalizumab: a review from the Research on Adverse Drug Events and Reports (RADAR) Project. Lancet Oncol 2009;10:816-24.

12. Stasi R. Rituximab in autoimmune hematologic diseases: not just a matter of B cells. Semin Hematol 2010;47:170-9. 